\title{
Análise morfométrica da bacia hidrográfica do rio Piranhas-Açú: trecho do estado do Rio Grande do Norte
}

Este trabalho teve como objetivo gerar as componentes para análise morfométrica da bacia hidrográfica do Rio Piranhas-Açu (BHRPA) no trecho do estado do Rio Grande do Norte, a partir de técnicas de geoprocessamento e sensoriamento remoto da superfície. Foram utilizados dados SRTM para elaboração do modelo digital de elevação (MDE). Elementos como fluxo de acumulação, sentido do fluxo, comprimento de fluxo entre outros foram obtidos pelo processamento no ambiente do software QGIS 2.18. Os resultados demonstraram que a BHRPA possui uma área total de $17.432 \mathrm{~km}^{2}$, perímetro de $817 \mathrm{~km}$, a rede de drenagem e moderadamente ramificada apresentando um comprimento total de $1.450 \mathrm{~km}$ com um curso principal de $245 \mathrm{~km}$. A bacia apresentou uma forma mais alongada, com densidade de drenagem inferior a $0,2 \mathrm{~km} / \mathrm{km} 2$, assim como baixa densidade hidrográfica. A classe de declividade predominante é do tipo plano a suave ondulada representado cerca de $40 \%$ da área total da bacia. As classes de coberturas compostas por pastagem natural, caatinga arbustiva com áreas agrícolas que dominam a paisagem ocupando uma área de 9.540 e 5.650, respectivamente, o que representa mais de $80 \%$ da cobertura do solo da área de estudo. Diferentes formas de relevo foram identificadas na bacia hidrográfica com base no SRTM (DEM) de dados com $90 \mathrm{~m}$ de resolução espacial. Técnicas de geoprocessamento foram assim caracterizadas pela altíssima precisão do mapeamento e mensuração dos índices morfométricos e revelaram-se como uma ferramenta com grande potencial para gestão de bacias hidrográficas.

Palavras-chave: Rede de Drenagem; Modelo Digital de Elevação; Geoprocessamento.

\section{Morphometric analysis of the Piranhas-Açú basin: State of Rio Grande do Norte stretch}

\begin{abstract}
This work aimed to generate the components for the morphometric analysis of the Piranhas-Açu River basin in the Rio Grande do Norte state (BHRPA), using geoprocessing techniques and remote surface sensing. SRTM data were used to elaborate the digital elevation model (MDE), elements such as accumulation flow, flow direction, flow length and others were obtained by processing in the QGIS 2.18 software environment. The results showed that the BHRPA has a total area of $17.432 \mathrm{~km}^{2}$ and perimeter of $817 \mathrm{~km}$, the drainage network is moderately branched, with a total length of $1.450 \mathrm{~km}$ with a main course of $245 \mathrm{~km}$, the basin presented a more elongated shape, with density of drainage less than $0.2 \mathrm{~km} / \mathrm{km} 2$ as well as low hydrographic density, the predominant declivity class is the flat to soft corrugated type represented about $40 \%$ of the total area of the basin. Covering classes composed of natural pasture, shrub caatinga with agricultural areas dominate the landscape occupying an area of 9.540 and 5.650, respectively, which represents more than $80 \%$ of the ground cover of the study area. Different relie forms were identified in the basin based on SRTM (DEM) data with $90 \mathrm{~m}$ spatial resolution. Geoprocessing techniques were thus characterized by the very high precision of the mapping and measurement of morphometric indices and proved to be a tool with great potential for watershed management.
\end{abstract}

Keywords: Drainage Network; Elevation Digital Model; Geoprocessing.

Pedro Vieira de Azevedo (iD Instituto Federal do Rio Grande do Norte, Brasil http://lattes.cnpq.br/0324478957720406 http://orcid.org/0000-0002-3136-5219 pvdeazevedo@gmail.com

Madson Tavares Silva (10)

Universidade Federal de Campina Grande, Brasil http://lattes.cnpq.br/8829792848536805 http://orcid.org/0000-0003-1823-2742 madson.geotech@gmail.com

Edivaldo Afonso de Oliveira Serrão (ic Universidade Federal de Campina Grande, Brasil http://lattes.cnpq.br/5700978243198274 http://orcid.org/0000-0003-2376-847X oliveiraserrao@gmail.com

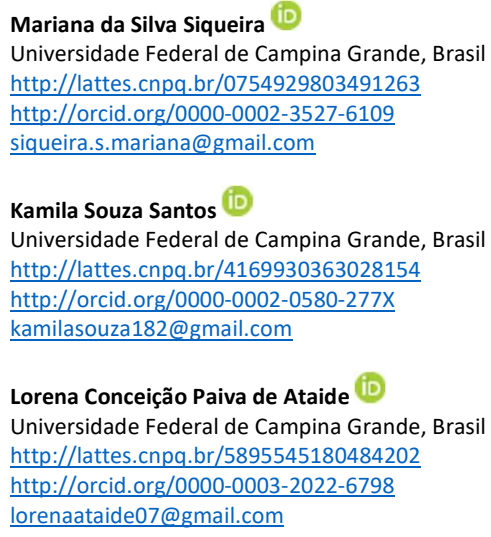

lorenaataide07@gmail.com

Leydson Galvíncio Dantas (iD

Universidade Federal de Campina Grande, Brasil http://lattes.cnpq.br/6496208673869879 http://orcid.org/0000-0003-4175-0951 leydsongalvincio@gmail.com SANTOS, K. S.; ATAIDE, L. C. P.; DANTAS, L. G.. Análise morfométrica da bacia hidrográfica do rio Piranhas-Açú: trecho do estado do Rio Grande do Norte. Revista Ibero Americana de Ciências Ambientais, v.11, n.2, p.434-444, 2020. DOI: http://doi.org/10.6008/CBPC21796858.2020 .002 .0039 


\section{INTRODUÇÃO}

A análise morfométrica representa uma medição e análise matemática da configuração da superfície terrestre, descrevendo assim a forma e a dimensão de seus acidentes geográficos. Uma grande ênfase na geomorfologia ao longo das últimas décadas provém do desenvolvimento de métodos quantitativos fisiográficos para descrever a evolução e/ou comportamento das redes de drenagem da superfície (PARETA, 2011). A origem da formação da rede drenagem de bacias hidrográficas tem sido discutida por diversos autores que baseiam seus estudos predominantemente nos componentes do escoamento superficial fluvial, com base nos fatores climáticos e na composição geológica seguida de efeitos biológicos (PARETA, 2011).

As características morfométricas das bacias hidrográficas podem conter informações importantes quanto à sua formação e desenvolvimento, uma vez que todos os processos hidrológicos e geomorfológicos ocorrem dentro da bacia. A análise morfométrica de uma bacia hidrográfica fornece uma descrição quantitativa do sistema de drenagem, o que é um aspecto importante para a caracterização de bacias hidrográficas (STRAHLER, 1952). Atualmente, o uso de técnicas de geoprocessamento tornou-se uma ferramenta amplamente utilizada para avaliar vários parâmetros do terreno e da morfometria das bacias de drenagem, uma vez que proporcionam um ambiente flexível para a manipulação e análise da informação espacial. Tais análises permitem a atualização da bacia de drenagem, ou ainda, a geração de informações para bacias pouco estudadas. $\mathrm{O}$ conhecimento da rede de drenagem das bacias hidrográficas é importante em qualquer processo hidrológico, pois possibilita a avaliação do potencial e gestão de águas subterrâneas (SILVA et al., 2015; SILVA et al., 2016; SILVA et al., 2017; SILVA et al., 2018; SERRÃO et al., 2019a; SERRÃO et al., 2019b)

Vários fenômenos hidrológicos podem ser correlacionados com as características fisiográficas de bacias hidrográficas como: tamanho, forma, inclinação da área de drenagem, densidade de drenagem e comprimento dos afluentes (RASTOGI et al., 1976).

Com o advento de sensores orbitais, modelos de elevação, que permitem a determinação da rede de drenagem puderam ser obtidos através da interferometria (SILVA et al., 2014; BARBOSA et al., 2017). Este processo é o modo de operação de alguns sensores imageadores de micro-ondas, como o exemplo do SRTM - Shuttle Radar Topographic Mission, ou da estereoscopia, através de sensores ópticos que adquirem imagens com retrovisada, a exemplo do ASTER/Terra - Advanced Spacebone Thermal Emission and Reflection Radiometer (FUCKNER et al., 2009).

O SRTM foi lançado para adquirir dados de altimetria de todo o globo terrestre, a partir de sensores ativos (radar). A resolução original das imagens geradas é de 30 metros; porém, para a América do Sul a NASA liberou imagens com resolução de apenas 90 metros. Os dados ASTER GDEM são produzidos através de um consorcio entre o METI - Ministry of Economy, Trade and Industry, do Japão e a NASA - National Aeronautics and Space Administration, dos Estados Unidos, para construção de uma modelo digital de elevação global de livre acesso. A partir de 29 de junho de 2009, modelos digitais de elevação, construídos a partir de pares estereoscópicos de imagens oriundas da plataforma EOS AM-1 com o instrumento ASTER, sensor VNIR, foram 
disponibilizados gratuitamente e sem restrições (RODRIGUES et al., 2010).

Dados de sensores remotos podem ser utilizados em conjunto com dados convencionais para delimitação dos cursos dos rios, caracterização da ordem, avaliação de prioridade de uso, avaliação e gestão de potenciais necessidades, identificação de áreas propensas à erosão, otimizando a tomada de decisões estratégicas de conservação da água, como também de seleção de locais para a construção de barragens e reservatórios (DUTTA et al., 2002).

Ramu et al. (2013), em estudo da morfometria da bacia de drenagem do rio Tungabhadra na Índia utilizaram dados SRTM. Rao et al. (2009a), utilizando técnicas de análise morfométrica demonstraram a dinâmica de equilíbrio que tem sido alcançado devido à interação entre matéria e energia para entender as características geo-hidrológicas das bacias de drenagem. Em análise mais aprofundada, Somashekar et al. (2011) para avaliar as características geo-hidrológicas de quatro sub-bacias hidrográficas do distrito de Agra na Îndia utilizaram técnicas de sensoriamento remoto e SIG. No Brasil algumas pesquisas utilizaram a técnica de análise morfométrica para caracterização de bacias hidrográficas em várias regiões do país (SILVA et al., 2012; ALVES et al., 2014; FERRARI et al., 2014; LOPES et al., 2018; CASSETTARI et al., 2019; SANTOS et al., 2019).

Assim, o presente trabalho teve como objetivo principal a determinação das características morfométricas e fisiográficas da bacia de drenagem do Rio Piranhas-Açú - BHRPA no estado do Rio Grande do Norte, visando contribuir com os estudos sobre planejamentos e gestão de bacias hidrográficas em regiões semiáridas.

\section{MATERIAIS E MÉTODOS}

\section{Área de estudo}

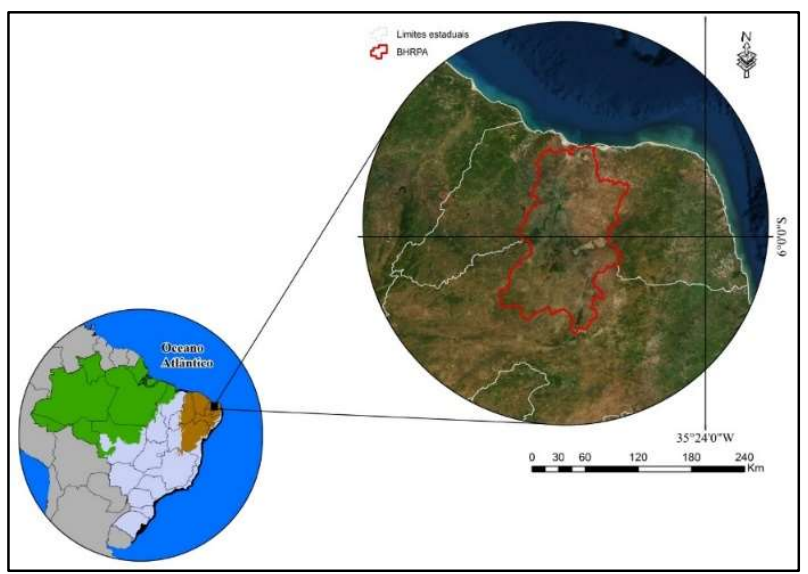

Figura 1: Localização da Bacia Hidrográfica do Rio Piranhas-Açú - BHRPA no estado do Rio Grande do Norte.

O presente trabalho foi realizado apenas nos domínios do estado do Rio Grande do Norte (RN) que detêm cerca de (40\%) da área total da Bacia Hidrográfica do Rio Piranhas - Açu (BHRPA) - Figura 1. A BHRPA é a maior unidade da região hidrográfica do atlântico nordeste oriental, correspondendo a $15 \%$ de sua área total, o que equivale a uma área de drenagem de $43.681,50 \mathrm{~km}^{2}$ (MMA, 2006). A BHRPA tem sua nascente na serra de piancó no estado da Paraíba e desemboca próximo à cidade de Macau (RN). Essa bacia 
hidrográfica é composta por rios intermitentes em condições naturais e é perenizada por dois reservatórios de regularização: Coremas - Mãe d’Água, na Paraíba, e a barragem Armando Ribeiro Gonçalves, no RN. A BHRPA está totalmente inserida em território semiárido, com precipitações médias variando entre 400 e 800 $\mathrm{mm}$ anuais, concentradas entre os meses de fevereiro e maio (PRH, 2016).

\section{Modelo digital de elevação do terreno - MDE}

Foi utilizado o modelo digital de elevação (MDE) com células de 90 metros apresentando precisão vertical compatível com a escala de 1:100.000 para a extração automática da rede de drenagem e delimitação da BHRPA. Tal modelo foi obtido no site da EMBRAPA, Monitoramento por satélite (CNPM) como resultado do projeto Brasil em Relevo (MIRANDA, 2005).

\section{Mapa de uso e ocupação do solo}

As informações que subsidiaram esta avaliação foram basicamente do mapa de cobertura do solo na escala 1:5.000.000 (IBGE, 2014).

\section{Mapa de solos da região}

As informações que subsidiaram esta avaliação foram basicamente do mapa de solos na escala 1:5.000.000 (IBGE, 2004). Esse mapa temático, fruto da parceria entre a EMBRAPA e o IBGE, permitiu uma visualização espacial dos principais tipos de solos segundo as especificações e nomenclatura indicadas pelo Sistema Brasileiro de Classificação de Solos - SiBCS (EMBRAPA, 1999).

\section{Análise morfométrica da bacia hidrográfica}

Para a extração das redes de drenagem foi utilizado o SIG QGis 2.18, com a finalidade de gerar os 'mapas de direção e acumulação de fluxo'. Foi necessário primeiro converter o MDE para o formato GRID, para depois gerar os mapas contendo as direções de fluxo e o fluxo acumulado. O mapa de fluxo acumulado foi classificado e as drenagens acima de 1000 foram extraídas. O valor 1000 foi um limite arbitrário adotado nesse estudo que se refere ao número mínimo de células do terreno necessárias para gerar células de drenagem. O método empregado para delimitação automática do limite da BHRPA foi de acordo com a ordem da hierarquia fluvial, com base na proposta de classificação de Strahler (1952).

Tabela 1: Características morfométrica que foram avaliadas na BHRPA.

\begin{tabular}{|c|c|c|c|}
\hline Característica Morfométrica & Descrição & Equação & Fonte \\
\hline Dd - Densidade de drenagem & $\begin{array}{l}\text { Relação entre o comprimento da rede de drenagem }(\mathrm{Cr}) \text { e a área } \\
\text { da bacia (A) }\end{array}$ & $\begin{array}{l}\mathrm{Dd}=\mathrm{Cr} / \mathrm{A} \\
\mathrm{Eq} \cdot 1\end{array}$ & Horton (1945) \\
\hline Dh - Densidade hidrográfica & $\begin{array}{l}\text { Relação entre o número de segmentos de rios }(\mathrm{Nt}) \text { e a área da } \\
\text { bacia }(\mathrm{A})\end{array}$ & $\begin{array}{l}\mathrm{Dh}=\mathrm{Nt} / \mathrm{A} \\
\mathrm{Eq} .2\end{array}$ & Christofoletti (1969) \\
\hline T - Razão de textura & $\begin{array}{l}\text { Relação entre o número de segmentos de rios }(\mathrm{Nt}) \text { e o perímetro } \\
\text { da bacia }(\mathrm{P})\end{array}$ & $\begin{array}{l}\mathrm{T}=\mathrm{Nt} / \mathrm{P} \\
\mathrm{Eq} .3\end{array}$ & $\begin{array}{l}\text { França (1968); } \\
\text { Smith (1950) }\end{array}$ \\
\hline Kf - Fator de forma & $\begin{array}{l}\text { Relação entre a área da bacia }(\mathrm{A}) \text { e o comprimento do eixo da } \\
\text { bacia (L). }\end{array}$ & $\begin{array}{l}\mathrm{Kf}=\mathrm{A} / \mathrm{L}^{2} \\
\mathrm{Eq} .4\end{array}$ & Horton (1945) \\
\hline $\begin{array}{l}\text { Kc } \quad \text { Coeficiente de } \\
\text { compacidade }\end{array}$ & Relação entre o perímetro da bacia (P) e a área da bacia $(\mathrm{A})$. & $\begin{array}{l}\mathrm{Kc}=0,28(\mathrm{P} / \mathrm{VA}) \\
\text { Eq.5 }\end{array}$ & Lima (1969) \\
\hline IC - Índice de circularidade & Relação entre a área da bacia $(\mathrm{A})$ e o perímetro da bacia $(\mathrm{P})$. & $\mathrm{IC}=12,57\left(\mathrm{~A} / \mathrm{P}^{2}\right) \mathrm{Eq} .6$ & Müller (1953) \\
\hline
\end{tabular}


Posteriormente, utilizando recursos do SIG foram calculados alguns parâmetros físicos tais como: área, perímetro, comprimento das redes de drenagens, número de segmentos dos rios, os quais formaram à base para diversos cálculos para determinar alguns parâmetros físicos da bacia hidrográfica em estudo (Tabela 1).

\section{RESULTADOS E DISCUSSÃO}

Conforme a Tabela 2, a Bacia Hidrográfica do Rio Piranhas-Açú - BHRPA no estado do Rio Grande do Norte apresenta uma área de $17.432 \mathrm{~km}^{2}$, com perímetro total de $817 \mathrm{~km}$ e 90 segmentos de rios, formando uma rede de drenagem de $1.450 \mathrm{~km}$ e o seu rio principal possui uma extensão de $245 \mathrm{~km}$. Segundo Yadav et al. (2014) a definição dos parâmetros morfométricos de uma bacia hidrográfica são extremamente importantes para identificar uma variedade de características hidráulicas da bacia de drenagem, isto é, forma, estágio de fluxo, permeabilidade da rocha, grau de degradação da rede de drenagem, assim como ajuda a comparar com a distinção litológica.

Vários autores destacam (PAKHMODE et al., 2003; RAO, 2009b; KUMAR et al., 2011) que elementos como a inclinação da bacia, natureza de rochas e a duração da atividade erosiva podem estar associado ao grau de permeabilidade das rochas e vice-versa configurando assim o mosaico em que a rede de drenagem se configura. No presente estudo as características da rede de drenagem da bacia mostram que a densidade de drenagem foi de $0,1 \mathrm{~km} / \mathrm{km}^{2}$, segundo a classificação de Christofoletti (1969) e, a densidade de drenagem da bacia é baixa, pois é menor que $7,5 \mathrm{~km} / \mathrm{km}^{2}$. Já para Villela et al. (1975), esse índice pode variar de 0,5 $\mathrm{km} / \mathrm{km}^{2}$, em bacias com drenagem pobre, a $3,5 \mathrm{~km} / \mathrm{km}^{2}$ ou mais, nas bacias excepcionalmente bem drenadas, indicando, portanto, que a bacia em estudo apresenta baixa drenagem de acordo com essa classificação.

Tabela 2: Características dimensionais da rede de drenagem da Bacia Hidrográfica do Rio Piranhas-Açú - BHRPA no estado do Rio Grande do Norte.

\begin{tabular}{|l|l|}
\hline Parâmetros & Valores \\
\hline Área da bacia (A) & $17.432 \mathrm{~km}^{2}$ \\
\hline Perímetro da bacia (P) & $817 \mathrm{~km}$ \\
\hline Comprimento do eixo da bacia (L) & $209 \mathrm{~km}$ \\
\hline Comprimento do Rio principal (R) & $245 \mathrm{~km}$ \\
\hline Comprimento da rede de drenagem (Cr) & $1.450 \mathrm{~km}$ \\
\hline Número de segmentos de rios (Nt) & 90 \\
\hline Densidade de drenagem (Dd) & $0,1\left(\mathrm{~km}^{\prime} / \mathrm{km}^{2}\right)$ \\
\hline Padrão de drenagem & Dendrítico \\
\hline Razão de textura (T) & $0,11(\mathrm{~km})$ \\
\hline Fator de forma (Kf) & $0,4\left(\mathrm{~m}^{2} / \mathrm{m}\right)$ \\
\hline Coeficiente de compacidade (Kc) & $1,73\left(\mathrm{~m} / \mathrm{m}^{2}\right)$ \\
\hline Índice de circularidade (IC) & 0,33 \\
\hline
\end{tabular}

O coeficiente de compacidade encontrado para bacia foi maior do que $1(1,73)$ e o fator de forma (Kf) foi 0,4 (considerado baixo). Por esse resultado pode-se afirmar que a bacia de drenagem, em condições normais de precipitação, ou seja, excluindo-se eventos de intensidades anormais, é pouco susceptível a enchentes. O fator de forma é usado para prever a intensidade do fluxo de uma bacia hidrográfica (GREGORY et al., 1973; MAGESH et al., 2012). Assim, há uma indicação de que a bacia não possui forma circular, mas uma tendência de forma alongada (SANTOS, 2001). É então possível inferir que o Kf está diretamente 
associado a como será o comportamento da rede de drenagem diante a eventos extremos dentro dos canais, ou seja, eventos em que altos picos de fluxo são verificados em um curto espaço de tempo são caracterizados em bacias circulares e menor fluxo de pico para maior duração em bacias mais alongadas. Tal fato pode, ainda, ser comprovado pelo índice de circularidade, cujo valor foi de 0,33 . Os parâmetros supracitados estão fortemente correlacionados entre si e são usados para descrever a configuração da bacia (SONI, 2016).

Conforme se pode observar na Figura 2, o padrão de drenagem predominante nessa bacia é do tipo dendrítico, ou seja, bem ramificado, semelhante a uma árvore que se, desenvolve em terrenos com predomínio de rochas mais resistentes. Tal fato pode estar associado por uma composição predominante de litologia resistente e homogênea (WITHANAGE et al., 2014). De acordo com (GARDE, 2005) tais padrões são configurados em áreas com predomínio de rochas ígneas maciças ou em rochas metamorfoseadas complexas.

Segundo Oruonye et al. (2016) parâmetros superficiais do aspecto do relevo de uma bacia hidrográfica fornecem informações sobre características do processo de desnudação provocadas pelos agentes do intemperismo, bem como características morfológicas de um terreno (HADLEY et al., 1961). Os autores ainda relatam que a intensidade dos processos erosivos pode ser configurada a partir da análise dos parâmetros de declividade da bacia.

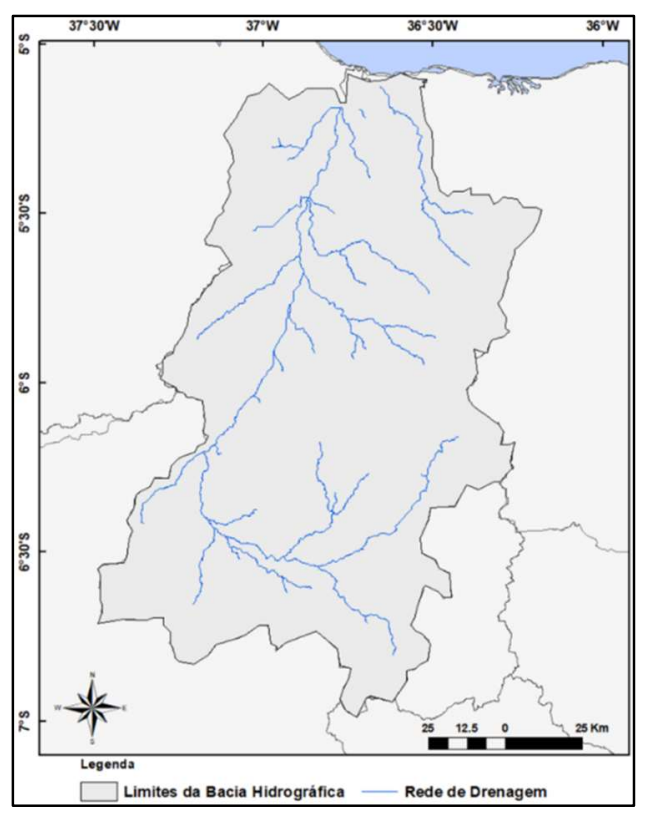

Figura 2: Rede de drenagem da Bacia Hidrográfica do Rio Piranhas-Açú - BHRPA no estado do Rio Grande do Norte.

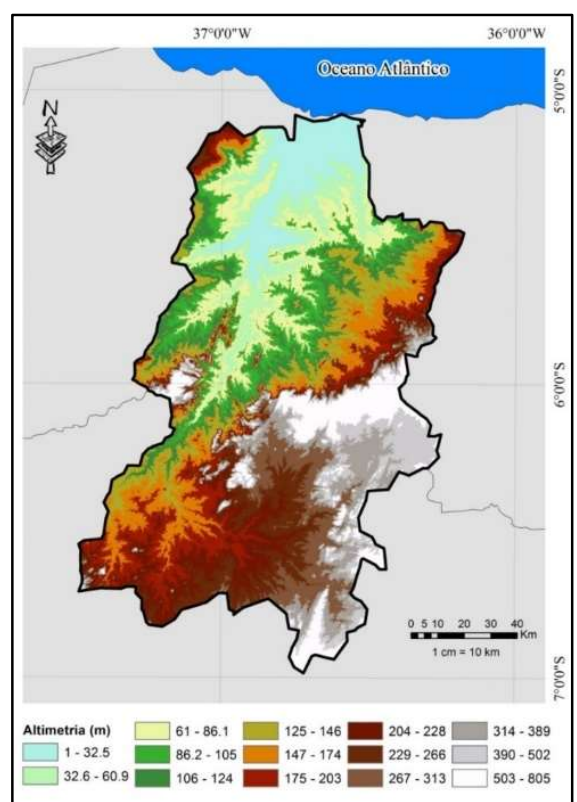

Figura 3: Mapa altimétrico da Bacia Hidrográfica do Rio Piranhas-Açú - BHRPA no estado do Rio Grande do Norte.

Sendo assim verificou-se para bacia de drenagem da BHRPA considerável variação de altitude (entre 1 e 805 m), com altitude média de 208 m e amplitude de 804 m (Figura 3), indicando que algumas regiões da bacia em questão favorece uma maior perda de água por escoamento superficial em função da rampa do declive. Áreas que apresentam altitudes inferiores aos 100 m correspondem a 4.642 km², ou 26,63\% do total, e localizam-se principalmente na parte noroeste. Em geral, o perfil hipsométrico caracteriza-se por altitudes inferiores ao $200 \mathrm{~m}$, que representam aproximadamente $60 \%\left(10.148 \mathrm{~km}^{2}\right)$ das superfícies da área de estudo. 
Trentin et al. (2005) relatam que o mapa hipsométrico tem fundamental importância na análise da energia do relevo, indicando condições mais propícias à dessecação para as áreas de maior altitude e de acumulação para as áreas de menor altitude. E assim quanto mais longo for o comprimento da bacia e mais íngreme a topografia, maior será o poder erosivo do escoamento.

De modo geral na bacia em análise mais da metade da área da bacia (68\%) apresentou declividades menores que $6 \%$, o que corresponde, segundo classificação de Biase (1993), o relevo de várzea e plano a suave ondulado, correspondendo aproximadamente a $12.000 \mathrm{~km}^{2}$. Todavia observou-se declividade superior a 55\%, em média a declividade a maior frequência de observações foi da ordem de 3\% (Figura 4). Essa classe compreende áreas com característica de suave ondulado a ondulado, onde a erosão hídrica não oferece problema e somente as práticas de conservação (preparo do solo e cultivo em nível) são necessárias, exceto em solos erodíveis (arenosos) com comprimento de rampa muito longo.

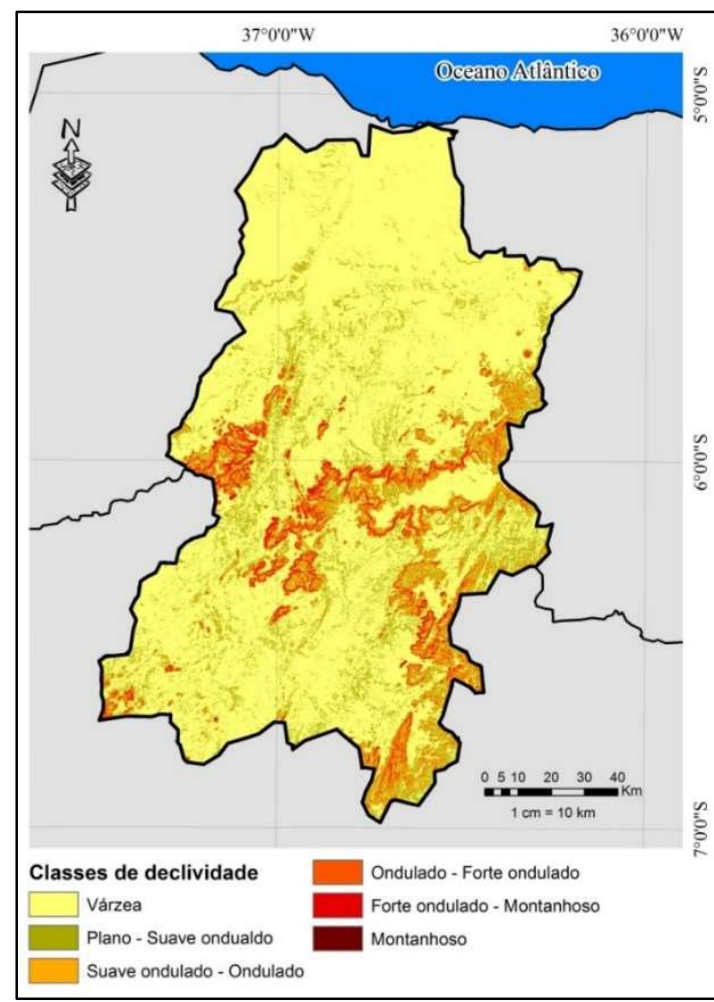

Figura 4: Mapa da declividade da Bacia Hidrográfica do Rio Piranhas-Açú - BHRPA no estado do Rio Grande do Norte.

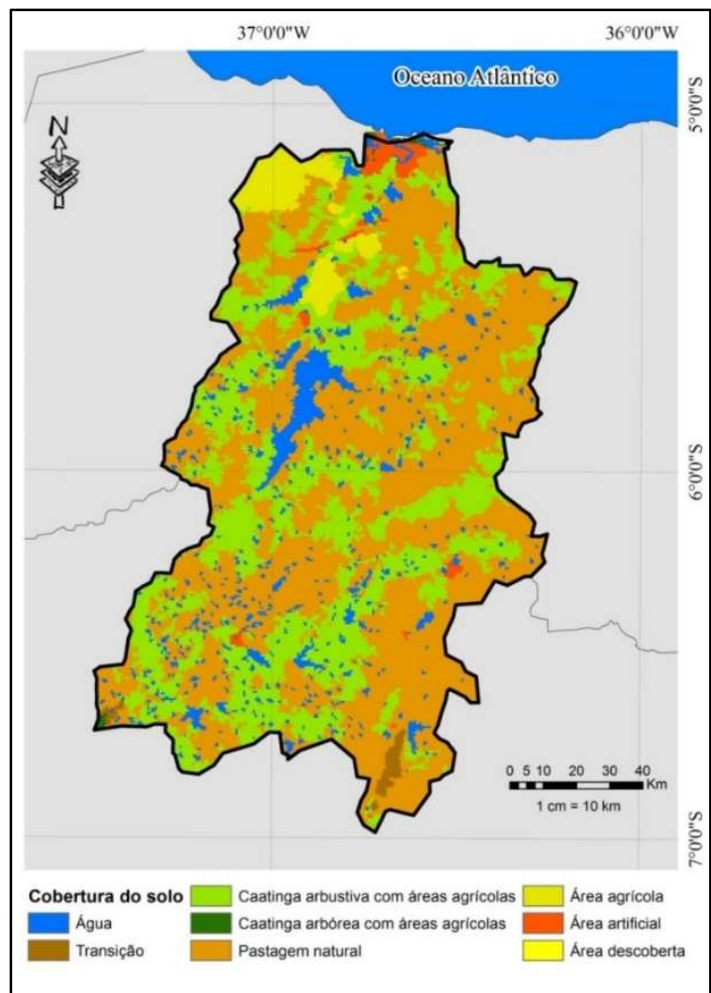

Figura 5: Mapa de uso e ocupação atual da Bacia Hidrográfica do Rio Piranhas-Açú - BHRPA no estado do Rio Grande do Norte.

Ainda de acordo com a Figura 3, destaca-se na parte central da bacia a incidência da classe "Forte ondulada - Montanhosa", que representa 5,8\% da área total, com abrangência de $1.000 \mathrm{~km}^{2}$. De acordo com Wilford et al. (2004) os valores dos parâmetros de declividades encontrados nessa pesquisa indicam que principalmente a parte central apresenta potencial para produção de sedimentos e transporte de massa para rede de drenagem, em detrimento da declividade mais íngreme. Tonello et al. (2006) remete que estudos de análise morfométrica contribuem na gestão dos recursos hídricos, principalmente no contexto da racionalização da água e não apenas para com o cumprimento da legislação ambiental. Ainda nessa perspectiva Guariz (2008) sugere que a degradação bem como a quantidade e qualidade de água disponível são resultantes de forçantes como o menor o tempo de concentração e aumento da velocidade do 
escoamento superficial resultando em menor a quantidade de água armazenada, o que amplia as perspectivas de enchentes mais pronunciadas. No entanto, a susceptibilidade da bacia à erosão severa do solo pode ser ainda mais modificada pela cobertura vegetal (Figura 5) e pelo tipo de solo (Figura 6).

E assim de acordo com a distribuição das classes de uso de solo identificadas na área em estudo (Figura 5), observa-se que a Pastagem natural e a Caatinga arbustiva com áreas agrícolas dominam a paisagem ocupando uma área, de 9.540 e $5.650 \mathrm{~km}^{2}$, respectivamente, totalizando cerca de 15.190 km², área correspondente a $87,14 \%$ da área total da bacia. O predomínio de áreas com vegetação nativa e cultivos intermite podem influenciar diretamente na recarga da água subterrânea da bacia. Ainda assim a capacidade de infiltração e a geração de escoamento podem ser influenciadas também pela frequência do fluxo dos rios, densidade de drenagem, e comprimento da bacia (SINGH et al., 2014; SONI, 2016).

Os cultivos irrigados (áreas agrícolas) são encontrados na parte norte da bacia, correspondente a $0.609 \mathrm{~km}^{2}$, representando menos de $4 \%$ da área total. Ainda de acordo com a (Figura 5) se verifica na parte central da bacia a presença da Barragem Engenheiro Armando Ribeiro Gonçalves - Barragem do Açú com capacidade de 2,4 bilhões de $\mathrm{m}^{3}$ de água (DNOCS, 2019) que contribui para a representação da classe de águas continentais da BHRPA, compreendendo 7,11\% da área total, com abrangência de 1.239 km².

Altaf et al. (2013) relata que a configuração da superfície da bacia hidrográfica no tocante das susceptibilidades à erosão além da inclinação acentuada da área e do tipo de cobertura do solo a presença de material subjacente menos resistente pode resultar em erosão severa do solo. Nesse contexto a BHRPA apresenta predomínio de três classes de solos (Figura 6), sendo aproximadamente $6.000 \mathrm{~km}^{2}$ ou $35 \%$ do total da área da bacia pertencentes à classe de Luvissolo Crômico, a classe de Neossolo Litólico representa pouco mais de $6.000 \mathrm{~km}^{2}$, a última classe de solo é a do Planossolo Nátrico apresentando 9,5\% da área total.

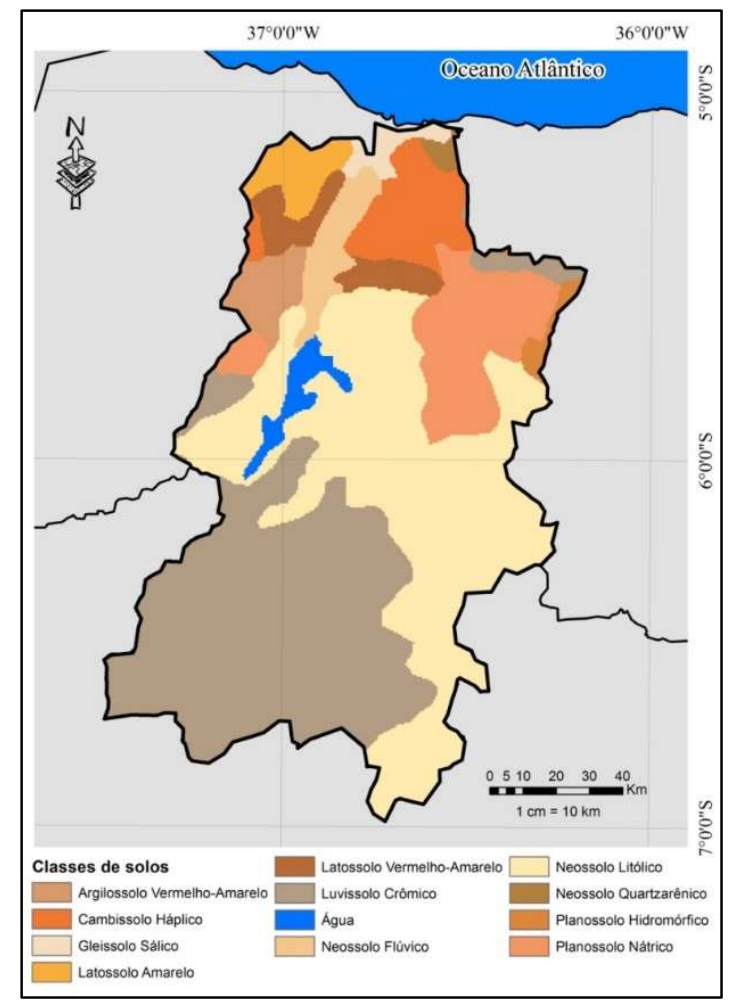

Figura 6: Mapa pedológico da Bacia Hidrográfica do Rio Piranhas-Açú - BHRPA no estado do Rio Grande do Norte. 
A caracterização pedológica da área de estudo é de grande importância tendo em vista que diferentes tipos de solo podem apresentar susceptibilidade diferenciada à erosão, mesmo para condições semelhantes de declividade, cobertura vegetal e práticas de manejo. Essas diferenças são devidas às propriedades do próprio solo e são denominadas erodibilidade do solo (BERTONI et al., 1985). Os mesmos autores classificam o fenômeno como sendo a vulnerabilidade do solo ou a sua susceptibilidade à erosão, que é a recíproca da sua resistência à erosão. As propriedades do solo que mais influenciam a sua erodibilidade são aquelas que afetam a taxa de infiltração da água no solo, associada à sua resistência ao cisalhamento.

\section{CONCLUSÕES}

Os resultados obtidos neste estudo revelaram a importância da utilização de dados obtidos por sensores remotos conjuntamente com plataformas SIG na abordagem baseada em avaliação de parâmetros morfométricos de drenagem e sua influência sobre formas de relevo, solos e características das superfícies.

Abordagens baseadas em SIG facilitam a análise de diferentes parâmetros morfométricos e a exploração da relação entre a morfometria de drenagem e propriedades de formas de relevo, solos e coberturas do solo. Diferentes formas de relevo foram identificadas na bacia hidrográfica com base no SRTM (DEM) de dados com $90 \mathrm{~m}$ de resolução espacial.

Técnicas de geoprocessamento foram assim caracterizadas pela precisão do mapeamento e mensuração dos índices morfométricos e se revelaram como uma ferramenta com grande potencial para gestão de bacias hidrográficas. A análise morfométrica da rede de drenagem da bacia hidrográfica indicou predominância do padrão dendrítico com baixa distribuição de segmentos de rios.

AGRADECIMENTOS: ao Conselho Nacional de Desenvolvimento Científico e Tecnológico (CNPq), pelo apoio financeiro a esta pesquisa (Processo $N^{\circ}$. 409499/2018-8) e pela concessão da bolsa de Professor Visitante no PPgUSRN/IFRN ao primeiro autor.

\section{REFERÊNCIAS}

ALTAF, F.; MERAJ, G.; ROMSHOO, A. S.. Morphometric analysis to inferhy- drological behavior of Lidder Watershed, Western Himalaya, India. Geography Journal, p.1-14, 2013. DOI: https://doi.org/10.1155/2013/178021

ALVES, T. L.; AZEVEDO, P. V.; SILVA, M. T.. Análise morfométrica da microbacia hidrográfico do riacho dos Namorados, São João do Cariri - PB: uma ferramenta ao diagnóstico físico. Revista de Geografia, v.31, n.3, p.130148, 2014.

BARBOSA, S. A. S.; JÚNIOR, A. J. S.; COSTA, G. J. A.; MIRANDA, S. M.. Uso de Modelo Digital de Elevação Aster Gdem e Srtm para Caracterização e Comparação de Rede de Drenagem. In: SIMPÓSIO REGIONAL DE GEOPROCESSAMENTO E SENSORIAMENTO REMOTO GEONORDESTE. Anais. Salvador, 2017. p.191-195.
BERTONI, J.; LOMBARDI, N. F.. Conservação do solo. 5 ed. São Paulo: Ícone, 2005.

BIASE, M.. A carta clinográfica: os métodos de representação e sua confecção. Revista de Geografia, v.6, p.45-60, 1993.

CASSETTARI, G. A.; QUEIROZ, T. M.. Morphometric characterization of the hydrographic basin of Jauquara river in the transition between the cerrado and amazon biomes in Mato Grosso-Brazil. Floresta, v.49, n.2, p.325-334, 2019.

CHRISTOFOLETTI, A.. Análise morfométrica das bacias hidrográficas. Notícia Geomorfologa, v.18, n.9, p.35-64, 1969.

DNOCS. Departamento Nacional de Obras Contra as Secas. Primeira reunião extraordinária da diretoria colegiada de 2019. DNOCS, 2019. 
DUTTA, D.; SHARMA, J. R.; ADIGA, S.. Watershed characterization, prioritization, development planningand monitoring remote sensing approach. Bangalore: ISRO, Technical report, 2002.

EMBRAPA. Centro Nacional de Pesquisa de Solos. Sistema brasileiro de classificação de solos. Brasília: Embrapa Produção de Informação; Rio de Janeiro: Embrapa Solos, 1999.

FRANÇA, G. V.. Interpretação fotográfica de bacias e de redes de drenagem aplicadas a solos da região de Piracicaba. Tese (Doutorado) - Universidade de São Paulo, São Paulo, 1968.

FERRARI, J. L.; SILVA, S. F.; SANTOS, A. R.; GARCIA, R. F. Análise morfométrica da sub-bacia hidrográfica do córrego Horizonte, Alegre, ES. Revista Brasileira de Ciências Agrárias, v.8, n.2, p.181-188, 2013

FUCKNER, M. A.; FLORENZANO, T. G. E.; MORAES, E. C. Avaliação Altimétrica de Modelos Digitais de Elevação Extraídos de Imagens ASTER em Áreas com Configuração Topográfica Distinta. In: SIMPÓSIO BRASILEIRO DE SENSORIAMENTO REMOTO, 14. Anais. Natal, 2009. p.25-30.

GARDE, R. J.. River morphology. New Delhi: New Age International Ltd., 2005.

GREGORY, K. J.; WALLING, D. E.. Drainage Basin Form and Process A Geomorphological Approach. London: Edward Arnold, 1973.

GUARIZ, H. R.. Morfometria e atributos físicos do solo da microbacia do córrego Jaqueira-Alegre, ES. Dissertação (Mestrado) - Universidade Federal do Espírito Santo, Alegre, 2008.

HADLEY, R. F.; SCHUMM, S. A.. Sediment sources and drainage Basin characteristics in upper Cheyenne River Basin. USGS Water-Supply Paper, v.1531, p.1-198, 1961.

HORTON, R. E.. Erosional development of streams and their drainage basins: hidrophysical approach to quantitative morphology. Bull. Geol. Soc. Am., v.56, p.275-370, 1945.

IBGE. Instituto Brasileiro de Geografia e Estatística. Mapa de solos do Brasil. Rio de Janeiro: IBGE, 2004.

IBGE. Instituto Brasileiro de Geografia e Estatística. Mapa de solos do Brasil: recursos naturais. Rio de Janeiro: IBGE, 2014.

KUMAR, A.; JAYAPPA, K.; DEEPIKA, B.. Prioritization of subbasins based on geomorphology and morphometric analysis using remote sensing and geographic informationsystem (GIS) techniques. Geocarto International, v.26, n.7, p.569592, 2011.

LIMA, W. P.. Manejo de Bacias Hidrográficas. Piracicaba: ESALQ, 1969.

LOPES, I.; LEAL, B. G.; RAMOS, C. M. C.. Morphometric characterization of the basin in the semiarid region of Pernambuco using SRTM data in free software. Journal of Hyperspectral Remote Sensing, v.8, p.31-40, 2018.
MAGESH, N. S.; JITHESHLAL, K. V.; CHANDRASEKAR, N.; JINI, K. V.. GIS based morphometric evaluation of Chimmini and Mupily watersheds, parts of Western Ghats, Thrissur District, Kerala, India. Earth Science Informatics, v.5, n.2, p.111-121, 2012.

MIRANDA, E. E.. Brasil em Relevo. Campinas: Embrapa Monitoramento por Satélite, 2005.

MÜLLER, V. C.. A quatitative geomorphic study of drainage basins characteristic in the Clinch Mountain area. Technical Report (3), Dept. Geology, Columbia University, 1953.

MMA. Ministério do Meio Ambiente. Caderno da Região Hidrográfica Atlântico Nordeste Oriental. Secretaria de Recursos Hídricos. Brasília: MMA, 2006.

ORUONYE, D. E.; EZEKIEL, B. B.; ATIKU, G. H.; BABA, E.; MUSA, I. N.. Drainage basin morphometric parameters of River Lamurde: Implication for hydrologic and geomorphic processes. Journal of Agriculture and Ecology Research International, v.5, n.2, p.1-11, 2016

PAKHMODE, V.; KULKARNI, H.; DEOLANKAR, S.. Hydrological drainage analysis in watershed programme planning - A case from the deccan basalt, India. Hydrogeol J., v.11, p.595-604, 2003.

PARETA, K.. Geo-Environmental and Geo-Hydrological Study of Rajghat Dam, Sagar (Madhya Pradesh) using Remote Sensing Techniques. International Journal of Scientific \& Engineering Research, v.2, n.8, p.1-8, 2011.

RAMU, B. M.; JAYASHREE, P.. Morphometric analysis of Tungabhadra drainage basin in Karnataka using GIS. Journal of Engineering, Computers \& Applied Science, v.2, n.7, p.17, 2013.

RAO, L. A. K.; REHMAN, Z.; SADIQUE, M.; ZAHOOR, I.. Hydrogeomorphological Studies for ground water prospect using IRS 1D, LISS-III image, in parts of Agra district along the Yamuna River. Jour. Environ. Res. Develop., v.3, n.4, p.12041210, 2009a.

RAO, N. S.. A numerical scheme for groundwater development in a watershed basin of basement terrain- $A$ case study from India. Hydrogeol J., v.17, n.2, p.379-396, 2009b.

RASTOGI, R. A.; SHARMA, T. C.. Quantitative analysis of drainage basin characteristics. Jour. Soil and water Conservation in India, v.26, n.1, p.18-25, 1976.

RODRIGUES, T. L.; BIASE, P.; SOUZA, R. F.. Avaliação da Adequação dos Produtos ASTER GDEM no Auxílio ao Mapeamento Sistemático Brasileiro. In: SIMPÓSIO BRASILEIRO DE CIÊNCIAS GEODÉSICAS E TECNOLOGIAS DA GEOINFORMAÇÃO, 3. Anais. Recife, 2010. p.1-5.

SANTOS, A. R.. Caracterização morfológica, hidrológica e ambiental da bacia hidrográfica do rio Turvo Sujo, microregião de Viçosa, MG. Tese (Doutorado) - Universidade Federal de Viçosa, Viçosa, 2001.

SANTOS, J. M.; SALAMUNI, E.; SILVA, C. L.; SANCHES, E.; GIMENEZ, V. B.; NASCIMENTO, E. R.. Morphotectonics in the 
Central-East Region of South Brazil: Implications for Catchments of the Lava-Tudo and Pelotas Rivers, State of Santa Catarina. Geomorphology, v.328, p.138-156, 2019.

SERRÃO, E. A. O.; SILVA, M. T.; SOUSA, F. A. S.; ATAIDE, L. C. P.; SANTOS, C. A.; SILVA, V. P. R.; SILVA, B. K. N.. Influência do uso e cobertura da terra na variabilidade espacial e temporal da evapotranspiração no sudeste da Amazônia, utilizando o modelo SWAT. Revista Ibero Americana de Ciências Ambientais, v.10, n.4, p.134-148, 2019a.

SERRÃO, E. A. O.; SILVA, M. T.; SOUSA, F. A. S.; LIMA, A. M. M.; SANTOS, C. A.; ATAIDE, L. C. P.; SILVA, V. P. R. Four decades of hydrological process simulation of the itacaiúnas river watershed, Southeast Amazon. Boletim de Ciências Geodésicas, v.25, n.3, 2019b.

SILVA, M. T.; AZEVEDO, P. V.; SILVA, V. P. R.; SILVA, B. K. N.; MARIANO, E. B.; AMORIN, M. R. B.. Estimate the sediment yield in the basin of lower-middle São Francisco River. Journal of Environmental Analysis and Progress, v.2, n.2, p.203-211, 2017.

SILVA, M. T.; SILVA, V. P. R.; SOUSA, E. P.; OLIVEIRA, S. D. Análise de Risco de Degradação na Bacia Hidrográfica do Riacho do Pontal - PE. Revista Brasileira de Geografia Física, v.3, p.646-660, 2012.

SILVA, M. T.; SILVA, V. P. R; SOUZA, E. P.; ARAÚJO, A. L. SWAT model application to estimate the flow in the basin of lower-middle São Francisco River. Revista Brasileira de Geografia Física, v.8, n.6, p.1615-1627, 2015.

SILVA, M. T.; SILVA, V. P. R.; SOUZA, E. P.; OLIVEIRA, V. G.. Performance analysis of digital elevation models: a case study of basin low middle São Francisco river. Journal of Hyperspectral Remote Sensing, v.4, n.7, p.161-167, 2014.

SILVA, V. P. R.; SILVA, M. T.; SINGH, V. P.; SOUZA, E. P.; BRAGA, C. C.; HOLANDA, R. M.; BRAGA, A. C. R.. Simulation of stream flow and hydrological response to land-cover changes in a tropical river basin. Catena, v.162, p.166-176, 2018.

SILVA, V. P. R.; SILVA, M. T.; SOUZA, E. P.. Influence of land use change on sediment yield: a case study of the submiddle of the São Francisco River basin. Engenharia Agrícola, v.36, n.6, p.1005-1015, 2016.
SINGH, P.; GUPTA, A.; SINGH, M.. Hydrological inferences from watershed analysis for water resourcemanagement using remote sensing and GIS- techniques. The Egyptian Journal of Remote Sensing and Space Sciences, v.17, p.111121, 2014.

SMITH, K. G.. Standars for grading texture of erosional topography. Am. J. Sci., v.248, p.655-668, 1950.

SOMASHEKAR, R. K.; RAVIKUMAR, P.. Runoff Estimation and Morphometric Analysis for Hesaraghatta Watershed, a remote sensing and GIS approach. Jour. Indian Soc. Remote Sensing, v.39, n.1, p.95-106, 2011.

SONI, S.. Assessment of morphometric characteristics of Chakrar watershed in Madhya Pradesh India using geospatial technique. App Water Sci. v.7, p.1-14, 2016.

STRAHLER, A. N.. Hypsometric analysis of erosional topography. Bull. Geol. Soc. Am., v.63, n.11, p.1117-1142, 1952.

TONELLO, K. C.; DIAS, H. C. T.; SOUZA, A. L.; RIBEIRO, C. A. A S.; LEITE, F. P.. Morfometria da bacia hidrográfica da Cachoeira das Pombas, Guanhães - MG. Revista Árvore, v.30, n.5, p.849-857, 2006.

TRENTIN, R.; ROBAINA, L. E. S.. Metodologia para mapeamento Geoambiental no Oeste do Rio Grande do Sul In: CONGRESSO BRASILEIRO DE GEOGRAFIA FÍSICA APLICADA, 11. Anais. São Paulo, 2005. p.3606-3615.

VILLELA, S. M.; MATTOS, A.. Hidrologia Aplicada. São Paulo: McGraw-Hill do Brasil, 1975.

WILFORD, D. J.; SAKALS, M. E.; INNES, J. L.; SIDLE, R. C.; BERGERUD, W. E.. RE- Cognition of debris flow, debris flood and flood hazard through watershed morphometrics. Landslides, v.1, p.61-66, 2004.

WITHANAGE, S. N.; DAYAWANSA, K. D. N.; SILVA, P. R. Morphometric analysis of the Gal Oya River Basin using spatial data derived from GIS. Tropical Agricultural Research, v.26, n.1, p.175-188, 2014.

YADAV, S. K.. Morphometric analysis of Upper Tons basin from Northern Foreland of Peninsular India using CARTOSAT satellite and GIS. Geocarto International, v.29, n.8, p.895914, 2014.

A CBPC - Companhia Brasileira de Produção Científica (CNPJ: 11.221.422/0001-03) detém os direitos materiais desta publicação. Os direitos referem-se à publicação do trabalho em qualquer parte do mundo, incluindo os direitos às renovações, expansões e disseminações da contribuiç̃o, bem como outros direitos subsidiários. Todos os trabalhos publicados eletronicamente poderão posteriormente ser publicados em coletâneas impressas sob coordenação da Sustenere Publishing, da Companhia Brasileira de Produção Científica e seus parceiros autorizados. Os (as) autores (as) preservam os direitos autorais, mas não têm permissão para a publicação da contribuição em outro meio, impresso ou digital, em português ou em tradução. 